\title{
SABER PEDAGÓGICO EN EVALUACIÓN DEL PROFESORADO DE ESCUELAS VULNERABLES DE LA COMUNA DE ARICAY SUS DEMANDAS A LA FORMACIÓN INICIAL DOCENTE*
}

\author{
PEDAGOGICAL KNOWLEDGE IN EVALUATION OF THE VULNERABLE \\ SCHOOL TEACHERS OF THE COMMUNITY OF ARICA AND ITS \\ CLAIMS TO THE INITIAL TEACHING TRAINING
}

\author{
Katherine Roberts-SÁnchez** \\ David Rodríguez-Gómez ${ }^{\text {**** }}$ \\ Patricia Silva*****
}

\section{Resumen}

Esta investigación tuvo como objetivo analizar las prácticas pedagógicas en evaluación del profesorado que se desempeña en contextos escolares vulnerables, identificando las demandas que, desde su experiencia profesional docente, realizan a la formación inicial. El estudio de carácter cualitativo, ha utilizado como técnica de recogida de la información, la entrevista semi estructurada y el grupo de discusión, y fue realizado en escuelas de alto índice de vulnerabilidad de la comuna de Arica-Chile, durante el primer semestre de 2018. Los resultados muestran que el profesorado utiliza estrategias evaluativas que promueven el diálogo, la reflexión grupal y la capacidad crítica del estudiantado, mediante procedimientos que incentivan la autoevaluación, la coevaluación y la metacognición de estos, estrategias que son complementadas con la formulación de preguntas

* Los resultados que se exponen, forman parte de la investigación para optar al grado de Doctor en Educación de la Universidad Autónoma de Barcelona. Barcelona, España, 2019

*** Doctora en Educación, profesora de artes visuales. Universidad Católica de la Santísima Concepción. Concepción, Chile, kroberts@ucsc.cl

**** Doctor en Educación, profesor de didáctica y organización escolar. Universidad Autónoma de Barcelona. Barcelona, España.

***** Doctora en Educación, profesora de didáctica y organización escolar. Universidad de Lleida, Barcelona, España. 
abiertas, la explicitación de altas expectativas sobre el rendimiento del alumnado y la utilización de recursos afectivos en su acción evaluativa.

Palabras clave: Evaluación del estudiante, práctica pedagógica en evaluación, estrategias educativas, formación de profesores, establecimientos de enseñanza vulnerables.

\begin{abstract}
The objective of this research was to analyze pedagogical practices in the evaluation of teachers working in vulnerable school contexts, identifying the demands that they make on initial teacher education from their professional experience. This is a qualitative study that used a semi-structured interview and a discussion group as techniques for data collection. It was carried out in schools with a high vulnerability index in the municipality of Arica, Chile, during the first semester of 2018. The results show that teachers use assessment strategies that promote dialogue, group reflection and the student's critical capacity, through procedures that encourage self-assessment, co-assessment and meta-cognition, all of which are complemented by the formulation of open questions, high expectations regarding student performance and the use of motivational resources in their assessment instances.
\end{abstract}

Keywords: Student assessment, Pedagogical practice in assessment, Educational strategies, Teacher education, Vulnerable teaching institutions.

\title{
1. Introducción
}

$\mathrm{H}$

ASTA AHORA Y MÁs ALlá del creciente interés del Estado Chileno por avanzar en el fortalecimiento de la calidad de la educación, con especial atención en aquellos estudiantes que asisten a escuelas vulnerables, no se han hecho esfuerzos serios por conocer, estudiar o valorar el saber pedagógico en evaluación de los docentes en general, ni menos de aquellos que se desempeñan en escuelas vulnerables. Esto porque como expresa Suárez (2017, p.47), la mayoría de los relatos del profesorado en ejercicio, no se conocen, se pierden o no se valoran, ya que sus experiencias “... son descalificadas como parte del conjunto de anécdotas triviales que los docentes usan para contarse entre sí lo que les pasa en las aulas y escuelas". 
Según Solar y Díaz (2018, p. 2), el saber pedagógico se construye "producto de la biografía y experiencia del docente, de sus conocimientos actuales y de su relación activa con la práctica”. Se erige en el transcurso de su vida como estudiante, en las prácticas pedagógicas contempladas en la malla curricular de su formación inicial, en el mundo laboral en que se desempeña, y en otras situaciones cotidianas como en las experiencias de cada persona y en sus relaciones sociales (López-Lozano, Solís y Fernández, 2017). Esto último, determinado por el contexto sociocultural donde el profesor está inmerso profesionalmente (Sotos y López, 2015).

Ideas similares han sido expresadas por Ibáñez (2014), al decir que el saber pedagógico es un saber teórico que origina el mismo educador a partir de la reflexión originada sobre su práctica docente y experiencia profesional. Y por Abraham (2017) en el sentido que el saber del profesorado, se establece a partir del quehacer pedagógico diario y se plasma en la teoría que construye el mismo profesorado, a partir de su experiencia y sus propios procesos de reflexión docente.

La revisión de la literatura (Espínola, Treviño, Guerrero y Martínez, 2017; Infante, Matus, Paulsen, Salazar y Vizcarra, 2013; Longás, Cussó, De Querol y Riera, 2016; Nanculeo y Merino, 2016; Traverso, 2016; Tucker, Trotman, y Martyn, 2015) reafirma la importancia de las particularidades y requerimientos específicos de los contextos escolares en los procesos de aprendizaje. De allí que, en el caso chileno, no sea suficiente conocer sólo el saber pedagógico en evaluación del profesorado, sino que, además, la importancia que concede al contexto escolar específico en que la ejerce, única manera de comprender el rol que corresponde a la evaluación y al profesor en estos procesos escolares.

Desde el inicio de la aplicación del Sistema de Evaluación Docente en Chile (2003), el Ministerio de Educación (MINEDUC) ha evaluado sobre el $83.3 \%$ del universo total del profesorado que, de acuerdo a procedimientos previamente fijados por la autoridad, deben someterse a esta evaluación. $\mathrm{El}$ propósito de esta iniciativa ha sido promover el desarrollo profesional docente y asegurar, el cumplimiento de los estándares de desempeño establecidos para la profesión a nivel nacional, cuyo propósito central es entregar a los niños y niñas una formación 
de calidad, mejorando sus aprendizajes (Docente Más / Sistema de Evaluación Docente de Chile, 2018).

Hasta el 2015, el portafolio contaba con las siguientes dimensiones a evaluar: organización de la unidad, análisis de la clase, calidad de la evaluación, reflexión a partir de los resultados de la evaluación, ambiente de la clase, estructura de la clase e interacción pedagógica. Luego del análisis de cada una de ellas, resulta interesante constatar cómo es que los ítems referidos a la calidad de la evaluación y reflexión a partir de los resultados de la evaluación, aparecen como las dos dimensiones en que los docentes evaluados obtienen una menor valoración.

En el primer caso, calidad de la evaluación, los resultados obtenidos por el profesorado estuvieron en torno a los 2.24 puntos, muy por debajo de los 2.5 puntos establecidos como el puntaje mínimo esperado de una escala de 1 a 4 puntos. Esta dimensión refiere la capacidad del profesorado para diseñar evaluaciones que se relacionan directamente con los objetivos de aprendizaje, presentar indicaciones o ítems claros y comprensibles para sus alumnos y utilizar pautas de corrección que identifican con precisión las respuestas o desempeños esperados (Docente Más / Sistema de Evaluación Docente de Chile, 2016).

En el segundo caso, reflexión a partir de los resultados de la evaluación, destinada a constatar la capacidad del profesor para entender cómo influyen sus propias decisiones pedagógicas, tanto en los aspectos logrados como no logrados de sus estudiantes, entregando una retroalimentación útil y específica para que estos mejoren sus aprendizajes, el puntaje alcanzó también un bajo resultado en relación a lo esperado, 2.17 de 4 puntos. (Docente Más / Sistema de Evaluación Docente de Chile, 2016).

Al poner en perspectiva los resultados obtenidos en uno y otro caso, resultan muy preocupantes, porque dejan en evidencia que el profesorado chileno evaluado tiene habilidades descendidas; al momento de diseñar y aplicar evaluaciones coherentes con los objetivos de aprendizaje, utilizar pautas de corrección que identifican con precisión los desempeños esperados y tomar decisiones a partir de los resultados de la evaluación, lo que dificulta el rol que corresponde a la evaluación en el propio aprendizaje, ya que como señala Gairín y Sanmartí (2010), de ésta depende tanto qué y cómo se enseña, como qué y cómo se aprende. 
Los resultados también reportan, cómo es que el profesorado a nivel nacional tiene dificultades para reflexionar acerca de cuánto influyen sus decisiones pedagógicas en los logros de sus estudiantes y en la importancia que posee una retroalimentación útil, oportuna y específica para que estos mejoren sus aprendizajes. Lo anterior hace que los estudiantes, si bien reciben los resultados cuantitativos de sus evaluaciones, no cuenten con una retroalimentación que les permita mejorar su desempeño, ni tampoco identificar con claridad cuáles fueron sus errores, ni menos el nivel de logro esperado por el profesor. Por lo que -tal como señala Moreno (2017) - se priva al alumnado de aprender, comprender y dialogar de manera óptima con su profesor en torno a sus procesos y resultados de aprendizaje.

Pero nuestras preocupaciones, respecto a la calidad de la evaluación realizada por el profesorado y a los procesos de reflexión efectuados a partir de los resultados de la misma, no se limitan solo a estas importantes cuestiones. Esto porque al examinar los resultados obtenidos por el profesorado, existe una variable que, a nuestro juicio, no ha sido lo suficientemente considerada, a pesar de haber sido relevada por la literatura (Gómez y Rivas, 2017; Sánchez, Díaz, Fuentes y Osorio, 2017; Sotos y López, 2015). Nos referimos al rol que juegan en la evaluación, la consideración de los contextos escolares, en los cuales el profesorado realiza cotidianamente su labor profesional.

Esta situación nos hace plantearnos que, si bien, la política pública chilena pretende promover el desarrollo profesional docente, este proceso se circunscribe mayoritariamente al desarrollo del propio quehacer y no contempla el abordaje de otros factores y variables que influyen en el aprendizaje de los estudiantes, especialmente referidos a sus diversos contextos de realización.

La falta de consideración de diversas realidades escolares en la formación inicial docente y la incipiente vinculación de los futuros profesores con el contexto escolar, que se ve refrendada en el informe de la Organización para la Cooperación y el Desarrollo Económico (OCDE , 2011), que -a propósito de la formación inicial- señala que esta carece de una vinculación más estrecha con la enseñanza en el contexto donde se realiza. Lo que, en el caso de nuestro país y en el espacio geográfico específico en el cual se realizó este estudio, adquiere 
gran significancia, por tratarse de una investigación que aborda los temas evaluativos, en un contexto escolar vulnerable.

En el caso de Chile, las escuelas vulnerables son establecimientos educacionales públicos, financiados por el Estado. Reciben alumnos en condición de extrema pobreza, que son categorizados anualmente por el MINEDUC; según el bajo nivel socioeconómico de las familias, el escaso nivel educativo de los padres y el riesgo de fracaso escolar al que están expuestos sus estudiantes. Por su parte, la Junta Nacional de Auxilio Escolar y Becas (2005, p. 14) define vulnerabilidad como la " interacción de una multiplicidad de factores de riesgo y protectores, que se manifiestan en conductas o hechos de mayor o menor riesgo social, económico, psicológico, cultural, ambiental y/o biológico, produciendo una desventaja comparativa entre sujetos, familias y/o comunidades".

En el ámbito escolar, la vulnerabilidad se entiende como una condición multidimensional de fragilidad de un estudiante y/o de una comunidad escolar en particular, que lo/s expone a factores de riesgo, propios de ese contexto, y que podrían afectar su capacidad de alcanzar logros académicos (Ñanculeo, 2014). En atención a lo anterior, si las condiciones de vulnerabilidad pueden ser similares para los estudiantes, pero los factores de riesgo en específico, responden a situaciones, características y requerimientos propios de los contextos escolares, entonces cabe preguntarnos ¿qué particularidades tienen las prácticas evaluativas de los profesores que trabajan en escuelas vulnerables?

\section{Diseño metodológico}

La investigación que se llevó a cabo es de carácter cualitativa (Dorio, Sabariego y Massot, 2016). Tuvo como propósito adentrarse en el mundo personal de quienes fueron estudiados y perseguir consenso entre los distintos significados, utilizando como criterio el acuerdo intersubjetivo en el contexto educativo (Sabino, 2014). La investigación recogió información particular desde la experiencia de los sujetos entrevistados, considerando el contexto donde se desempeñan profesionalmente para generar saberes desde las perspectivas de los profesores participantes. 
El diseño de investigación fue de tipo descriptivo, dado que su propósito fue observar fenómenos tal como se dan en su contexto natural, para su análisis posterior (Hernández, Fernández y Baptista, 2010).

El método de investigación, entendido por López y Sandoval (2016), como la forma característica de investigar, determinada por la intención y enfoque que la orienta, fue la Teoría Fundamentada (TF), ya que los procedimientos de indagación orientaron a los investigadores para construir teorías sobre los entornos y la vida de los individuos, con criterios de rigor metodológico y de compatibilidad entre teoría y observación, perdurables en su alcance teórico (Strauss y Corbin, 2002).

En la TF, la teoría se vuelve indispensable para el conocimiento profundo de los fenómenos sociales y constituye la mejor forma para representar la realidad social, por cuanto la teoría surge-inductivamente de los datos (Bonilla y López, 2016). En la TF, los datos constituyen categorías preliminares, luego códigos conceptuales y posteriormente, conceptos centrales o teoría sustantiva.

\section{Procedimientos de recogida y análisis de la información}

Los instrumentos seleccionados para la recogida de la información fueron la entrevista semi-estructurada y el grupo de discusión. Fue elegida la entrevista semi-estructurada porque de acuerdo con Hernández, Fernández y Baptista (2010), ésta se caracteriza por ser íntima y flexible y porque además ofrece la oportunidad de realizar una reunión para conversar e intercambiar información entre el entrevistador y el entrevistado, lo que facilita la recogida de la información. La selección del grupo de discusión se explica porque -como señalanDorio, Sabariego y Massot, (2016) permite que los discursos de los participantes en el grupo "choquen" entre sí y se escuchen hasta llegar a consensos.

Glaser y Strauss (2009) proponen que las estrategias fundamentales de la TF son el Método Comparativo Constante (MCC) y el muestreo teórico, debido a que estas estrategias vinculan constantemente la recogida de la información, su codificación y el análisis de los 
datos, lo que hace que la recogida de la información y el análisis de la misma se desarrolle de forma paralela y constante. De esta manera, el estudio de la información no es lineal sino circular, ya que el investigador analiza los datos y levanta la teoría en un ir y venir durante la revisión de los datos.

El corpus de conocimiento estuvo compuesto por una masa textual aproximada de 45.000 palabras, y fue analizado en el programa Atlas ti. La organización de la generación de datos o proceso de codificación se realizó en tres etapas: codificación abierta, codificación axial y codificación selectiva (Strauss, Corbin y Zimmerman, 2002).

La codificación abierta fue entendida como aquel "proceso de abordar el texto, con el fin de desnudar conceptos, ideas y sentidos" (San Martín, 2014, p. 110). Incluyó el análisis del texto, producto de la entrevista con el sujeto investigado, con el propósito de descubrir las ideas, pensamientos y significados contenidos en él. Esta primera etapa de análisis fue un proceso riguroso e inductivo que requirió del examen meticuloso de los datos para identificar y conceptualizar sus significados, prescindiendo de la teoría formal para su codificación.

Después de la codificación abierta, los códigos provenientes de esa etapa fueron reorganizados en categorías conceptuales de nivel mayor de abstracción, lo que responde a la etapa siguiente de codificación axial o etapa de integración de varias categorías abiertas. A través de este procedimiento, se crean nuevas combinaciones de códigos con miras a formar las categorías y subcategorías, que, a su vez, fueron organizadas en coherencia con los relatos de los hechos en la escena social. En esta segunda etapa, “... las intuiciones iniciales respecto de la relación entre categorías y subcategorías se expresan en hipótesis que explican el qué, por qué, dónde y cómo de un fenómeno" (San Martín, 2014, p. 111).

La codificación selectiva o teórica fue el tercer nivel de codificación. Tuvo como objetivo refinar e integrar categorías y subcategorías, exponiendo aquellas categorías consideradas centrales, por contener a todas las demás, y que se convierte en la teoría emergente del estudio. $\mathrm{Al}$ respecto, Flick (2018, p. 198) señala que la codificación selectiva permite "elaborar la categoría central en torno a la cual las otras categorías desarrolladas se pueden agrupar y por la cual se integren. De esta forma, se elabora o formula el relato del caso". 


\section{Muestra}

El muestreo teórico o la determinación del número de sujetos informantes no fue definido a priori, sino que se fue ajustando durante el avance de la recogida de información, en coherencia con el criterio de saturación teórica definido por la TF. En este estudio, la saturación se evidenció en el momento en que los relatos aportados por el profesorado y el análisis ulterior de las categorías, no evidenciaban propiedades nuevas, constituyéndose en categorías centrales de análisis o códigos teóricos (Monge, 2015).

La muestra inicial fue conformada por ocho profesores y luego del análisis preliminar de la información aportada por ellos en las entrevistas y siguiendo la estrategia de la TF, de incorporar un nuevo sujeto según su potencial para ampliar y profundizar los conceptos ya desarrollados (Jiménez-Fontana, García-González, Azcárate, Navarrete y Cardeñoso, 2016), se amplió el muestreo hasta llegar a dieciséis profesores entrevistados (11 mujeres y 5 hombres), pertenecientes a siete establecimientos educacionales, de dependencia municipal, ubicados en la Comuna de Arica, con quienes se cumplió la saturación teórica. Complementariamente, la selección de los profesores participantes en el grupo de discusión, se realizó de acuerdo a los mismos requisitos de los educadores que conformaron la muestra teórica inicial y fue constituida por ocho profesores.

Los profesores investigados fueron seleccionados intencionadamente. Los criterios de discriminación fueron los siguientes: i) poseer un mínimo de cinco años de ejercicio profesional en aula; ii) enseñar más de una materia en el ciclo básico de formación escolar $\left(1^{\circ}\right.$ a $8^{\circ}$ básico en Chile) y iii) desempeñarse laboralmente, al menos, durante dos años en la misma escuela de la comuna de Arica. En relación con la elección de los establecimientos escolares, debían disponer de un alto índice de vulnerabilidad escolar, esto es, establecimientos educacionales que reciben estudiantes en condición de extrema pobreza. 


\section{Resultados}

En concordancia con las preguntas centrales y los objetivos trazados en el estudio, los resultados obtenidos dan cuenta del camino recorrido por el profesorado en la construcción de su actual saber pedagógico en evaluación, siendo agrupados en dos grandes categorías: estrategias evaluativas utilizadas por el profesorado que desempeña en escuela que poseen estudiantes en condición de vulnerabilidad y demandas que, desde su experiencia, realizan a la formación inicial del profesorado. La primera, explica las características que posee la evaluación impartida por el profesorado en establecimientos vulnerables, procurando identificar algunas de las estrategias empleadas por los mismos en lo que llaman la transición desde la evaluación tradicional a la auténtica. La segunda da cuenta de los requerimientos que sobre evaluación realiza el profesorado a la formación inicial de profesores, si la pretensión fuera que sus egresados se desempeñen con alguna soltura en contextos vulnerables.

\subsection{Estrategias evaluativas utilizadas por el profesorado}

Las ideas expresadas por el profesorado coinciden en señalar que la manera en que ellos aplican los instrumentos y procedimientos de evaluación (por ejemplo, trabajos grupales, trabajos prácticos, disertaciones y dramatizaciones), incentiva la reflexión grupal y personal, con especial énfasis en desarrollar la capacidad de crítica del estudiantado frente a su propio trabajo personal y el de sus compañeros.

El profesorado expresa que, a través de la coevaluación y la autoevaluación, el estudiantado aprende a opinar críticamente sobre sus propios resultados y sobre el desempeño colectivo de sus pares. Además, son capaces de identificar los aspectos positivos y mejorables de cada resultado de aprendizaje obtenido, valorando los recursos afectivos utilizados por el profesorado, los que actúan como un poderoso estímulo para realizar este análisis. Una muestra de los beneficios de la evaluación en el desarrollo personal de los estudiantes, es el que se cita a continuación: 
... primero la parte crítica, porque ellos aprenden en mi asignatura a ser como críticos, lo otro a compartir digamos de sus propios conocimientos, porque trabajamos en grupo, por ejemplo, ellos aprenden una cosa del otro, y comparten y ahí ellos aportan, y lo otro que ayuda mucho, que ellos sean capaces, que vayan aprendiendo también a conocerse a quererse, y es increíble que eso les ayuda para tener buenas evaluaciones. (Entrevista 3)

El profesorado también reconoce que el tipo de preguntas realizadas a los estudiantes (de respuesta abierta aplicadas de manera intencional), favorecen la autocrítica del estudiantado y su disposición al aprendizaje y la reflexión. Esto, porque les da la oportunidad de entrar a las respuestas desde diferentes perspectivas y ángulos, sintiéndose libres de ensayar una respuesta desde su propio discurso, porque saben que no se busca evaluar su capacidad de menor o mayor nivel de memorización, lo que, a largo plazo, también les permite aprender.

En el testimonio de los profesores, se expone la idea de estar muy atentos a promover la metacognición, entendida como la toma de conciencia sobre el conocimiento que tienen los sujetos sobre sus propios procesos mentales; qué saben y cómo aprenden (Monereo y Barberá, 2000). Al respecto, una profesora señala:

... me gusta cuando los niños reflexionan y ellos mismos dicen "profe sabe qué no me había percatado". Me pasa con geografía, cuando a los niños les digo: "oye ustedes viven en pleno desierto de Atacama, en el desierto más seco del mundo, vayan al agro y va a haber comida todo el año ¿por qué creen que pasa eso?... (Entrevista 6)

Con convicción y entusiasmo, el profesorado narra cómo es que sus prácticas evaluativas son una acción pedagógica favorecedora del diálogo; entre el profesor y los estudiantes, entre los estudiantes y los padres y/o apoderados y principalmente, entre los propios estudiantes, permitiendo que el estudiantado pueda compartir sus conocimientos, promover el trabajo colaborativo y apoyarse entre ellos.

En este contexto escolar, desde los relatos del profesorado, se aprecia con claridad el aprendizaje colaborativo, donde la colaboración 
surge de manera natural y espontánea. Lo que en palabras de Roselli (2016, p.223) se traduce como "un proceso colectivo desde el inicio, donde todos intervienen conjuntamente en la realización de la tarea". Mientras que en el contexto de nuestra investigación, el profesorado señala que: “... los niños lograban terminar [las tareas] con apoyo, algunos con más apoyo, vimos también que los chiquillos se apoyaban mutuamente, el que aprendió primero ayudaba al otro...". (Entrevista 12)

Con evidente orgullo, el profesorado releva sus acciones evaluativas como favorecedoras de aprendizaje, no sólo en el ámbito cognitivo, sino también en el procedimental y actitudinal, reconocido por los estudiantes, quienes advierten sus avances en sus habilidades de comunicación oral y expresión escrita. Al respecto, un profesor señala: “... a ellos les costaba por la vulnerabilidad social-cultural y por su origen [Aymará] un poco más expresarse, les era más fácil demostrar lo que habian entendido plasmado en el trabajo. Entonces, cuando tú llegabas y le preguntabas ¿explícame algo?, a ellos le costaba expresarlo..."(Entrevista 8).

Otra idea que destaca en el relato del profesorado, está relacionada con sus altas expectativas, para con el aprendizaje de sus estudiantes. Según ellos, lo anterior permite "romper" el círculo del "no puedo" y la negación hacia el aprendizaje, promoviendo una actitud positiva hacia él y particularmente hacia el rol que cumple la evaluación en este proceso. Existe una correlación positiva entre sus altas expectativas y los buenos resultados obtenidos por sus estudiantes, lo que fue recientemente ratificado una vez más por Murillo y Martínez-Garrido (2018, p.11), al decir que "se ha encontrado que los profesores que consideran que sus alumnos van a aprender determinado tema o van a alcanzar un nivel de estudios lo harán con mayor probabilidad”. Según el profesorado en estudio, sus altas expectativas se evidencian cuando les dicen reiteradamente a sus estudiantes expresiones como "todos pueden aprender", "ustedes son inteligentes y capaces de aprender esto y mucho más”, y "todos pueden lograr las metas que se propongan”.

Una nueva estrategia que destaca el profesorado, que también evidencia sus altas expectativas, tiene que ver con su consciente disposición a que todos los estudiantes tengan la oportunidad de participar en la clase y no sólo aquellos que espontánea y/o habitualmente lo hacen. Para ellos, es especialmente relevante que las preguntas que 
preparan para la clase puedan ser respondidas por la mayor parte del estudiantado. Esta distribución intencionada de la participación de los alumnos, hace que ellos se sientan partícipes de la clase, refuercen su capacidad para responder las interrogantes planteadas por el profesor y lo que es aún más interesante, puedan generar sus propias interrogantes sobre los tópicos de la clase. En palabras de los docentes, esta estrategia repercute positivamente en la autoestima y disposición de los estudiantes hacia el aprendizaje. El siguiente fragmento describe lo recién mencionado:

... yo creo que los niños cambiaron de actitud y lo que más nosotros valoramos es la efectividad, que los niños se sentían más empoderados, que se sentían satisfechos, que ellos podían lograr, yo siempre con el profe fomentamos mucho "ustedes pueden chiquillos", "ustedes pueden lograrlo", descubrimos cómo te digo otros talentos, entonces "eres fantástico haciendo una obra" de aquello que quizás otros dirían "aaah no sirve", pero nosotros no pensamos ni decimos eso... (Entrevista 8)

Otra idea, que el profesorado que se desempeña en colegios vulnerables promueve de manera consciente, es ejecutar su acción pedagógica, incorporando distintos recursos afectivos. Lo anterior hace que otorguen mucho valor al afecto como herramienta mediadora y generadora de diálogo con el estudiantado y entre ellos mismos. Esto se ha estudiado en la literatura (Martínez, 2015) con el nombre de recursos afectivos, urdimbre afectiva (Rof, 1975) o emociones (Casassus, 2017), definidas por Cubillos (2019, p. 84) como "un flujo de energía encarnada, de carácter relacional, que contiene información y que impulsa a la acción". Con ello quieren dar cuenta de la expresión constante de palabras motivantes y gestos afectuosos, por parte del profesorado, las que -a juzgar por los propios profesores- cumplen una función tranquilizadora, liberadora y ordenadora en los estudiantes. El siguiente fragmento expresa con claridad la aplicación de recursos afectivos en las prácticas evaluativas de los entrevistados:

Bueno y también usamos mucho la parte afectiva, ya entonces cuando uno dice "Que bien lo hiciste", "maravilloso", porque son niños que tú les dices eso y les sube el autoestima, sienten, se empoderan y vienen 
a la otra clase con “¿Profe qué vamos hacer hoy día?, ¿Qué trabajo va a dejar?", va cambiando la mentalidad de los niños, se van sintiendo reconocidos, pucha puros rojos y ahora soy capaz, puedo, les subes las expectativas de ellos que siempre están bajas, entonces es muy bonito... (Entrevista 11)

Lo anterior ha hecho que, en su desempeño profesional, el profesorado valore como parte inherente a la construcción de su saber pedagógico en evaluación, el desarrollo de sus propias habilidades emocionales, aprendiendo a ser más cercanos y empáticos en la relación con sus estudiantes. El saber pedagógico así construido les ha permitido incorporar un conjunto de recursos afectivos (como la amabilidad y la disposición consciente a escucharlos), en su quehacer cotidiano, que se traduce en clases más distendidas y cercanas y en la generación de interacciones más fluidas, menos frontales y centradas en la persona que aprende, quienes se sienten acogidos y comprometidos no solo cognitivamente por el profesorado, sino que también, y por sobre todo emocionalmente.

Lo anterior hace que el profesorado que se desempeña en contextos vulnerables, valore este proceso como parte importante de su derrotero profesional, cuya particularidad central desde el punto de vista evaluativo, es el de una transición entre una evaluación tradicional a una cada vez más auténtica. Esto porque la utilización de recursos afectivos como el diálogo fraterno, la empatía y el buen trato a los estudiantes, incorpora dimensiones formativas que no son posibles de evaluar bajo una concepción más tradicional. Al menos, así se evidencia en el siguiente relato:

Por lo general converso con él y le pregunto si pasó algo en casa, cuando el alumno abre diálogo yo voy más allá incluso, a veces lo derivo a orientación cuando ya el tema escapa de mi competencia, o bien, por lo general, los de mi jefatura yo cito al apoderado. Entonces, “¿mamita qué pasó?”"Él es súper cumplidor, es súper responsable, yo sé que usted viene, y que está bien al pendiente, pero en esta prueba está súper mal, entonces de repente, sucedió algún evento en la casa", me he encontrado con "el domingo mi papá llegó borracho a la casa y nos tuvimos que ir a dormir donde mi abuelita y no estudié nada... (Entrevista 6) 
Los recursos afectivos contribuyen a concebir la evaluación como un proceso que pretende no sólo una preocupación por la construcción de conocimientos por parte del estudiantado, sino que también -como veremos en el segundo código-, de habilidades y actitudes, lo que resulta coherente con las exigencias curriculares que posee el $\mathrm{Marco} \mathrm{Cu}^{-}$ rricular Nacional de Chile.

Pero la sola utilización de recursos afectivos no basta porque a renglón seguido el profesorado reconoce que el círculo se cierra en el momento en que informan los resultados de aprendizaje, espacio imprescindible en donde se informa al estudiantado (ver entrevista 3 ).

La incorporación de recursos afectivos en sus prácticas evaluativas, es concebida por el profesorado como un proceso que los hace sentir más humanos, cercanos y empáticos con las necesidades que poseen sus estudiantes, lo que -de acuerdo al relato del propio profesorado- el estudiante capitaliza en mayor confianza y como consecuencia, tributa en más y mejores aprendizajes. Así se desprende de los siguientes relatos: "... he aprendido a modificar mi estructura, mis evaluaciones, como te digo a ser más humana..." (Entrevista 7)

\subsection{Demandas del profesorado a la formación inicial}

Los requerimientos a la formación inicial de profesores dan cuenta de las sentidas demandas, en el ámbito de la evaluación, que propone el profesorado que se desempeña en contextos vulnerables, a las instituciones que forman futuros profesores. Los requerimientos surgen desde su propia experiencia docente y del saber pedagógico que han construido y acumulado en el contexto escolar donde se desempeñan. Con el propósito de hacer una presentación adecuada de las demandas, éstas se han agrupado en tres sub categorías: i) formar al profesorado para evaluar constantemente, ii) formar al profesorado para retroalimentar después de evaluar y iii) formar al profesorado para evaluar en diversidad de contextos.

En relación a la primera demanda, formar al profesorado para evaluar constantemente, el profesorado que participó en el estudio señala que las observaciones provienen desde su propia experiencia profesional en contexto de vulnerabilidad que han ido incorporado a través del 
tiempo en su formación profesional. El profesorado señala que, si bien en las instituciones de educación superior donde fueron formados se les enseñó a evaluar, este proceso tuvo como sustento estándares formativos tradicionales y homogéneos, que no dan cuenta de la diversidad existentes en sus aulas, y que gracias a su ejercicio profesional han logrado construir. Esta experiencia profesional les ha demostrado la necesidad de evaluar continuamente el desempeño de sus estudiantes, considerando el contexto y las necesidades particulares de los mismos y desestimando la idea tradicional, según la cual, se debe esperar al final de un proceso para hacerlo, sin considerar el contexto y las características personales de vulnerabilidad del alumnado. En otras palabras, y siguiendo una expresión dada por los propios entrevistados, la evaluación "debe considerar los contextos y estar al servicio de los estudiantes, pero, esto significa que hay que programar una evaluación para los estudiantes y con ellos". (Entrevista 5)

El profesorado también aboga con entusiasmo sobre la necesidad que en la formación inicial "se oriente la evaluación como un mecanismo que sirva para conocer el avance en el aprendizaje de los estudiantes, no sólo desde los ámbitos conceptuales, sino que también de aquellos aprendizajes procedimentales y principalmente actitudinales" (entrevista 3). Fruto de su experiencia en evaluación -agregan que la evaluación-, "promueva una concepción que por sobre todo releve la evaluación como una experiencia constante y variada de recogida de información, subrayando que este proceso sea coherente con las características particulares del contexto educativo y en consideración de las características y la realidad cotidiana del estudiantado" (entrevista 4). El siguiente relato da cuenta de la importancia que le dan a la evaluación de proceso: “... evalúo durante todas las clases, al final de cada clase, haciéndole preguntas a los niños; ¿lles quedó claro?, o ¿no quedó claro?, entonces vuelvo a evaluar al inicio de la próxima clase para activar los conocimientos previos". (Entrevista 11)

No obstante lo anterior, cuando al profesorado se insta a explicar en profundidad porqué es importante para ellos la evaluación formativa o de proceso, que no requiere asignar una calificación, aparecen tensiones entre la concepción evaluativa auténtica -a la que se declaran adscribir- y lo que realmente hacen en la sala de clases, que 
son prácticas evaluativas más cercanas a la evaluación tradicional. E1 siguiente fragmento, ejemplifica esa tensión:

Superviso la clase varias veces, al inicio de las clases, la fecha, la actividad que vamos a realizar, los objetivos, les explico qué va a pasar en la clase, ellos ya tienen puesta la fecha, entonces la primera supervisión mía que yo voy viendo en los cuadernos que esté la fecha registrada. (Entrevista 4)

Respecto a la segunda demanda efectuada por el profesorado, en torno a formar a los futuros profesores en una concepción evaluativa que valore la retroalimentación luego de la evaluar, éstos profundizan en la importancia de comunicar oportunamente a los estudiantes sus avances y resultados, con la clara intención de generar aprendizaje en ellos. Entre todos sus argumentos, puntualizan la necesidad de retroalimentarlos verbalmente, por escrito o gestualmente, tanto durante el proceso de aprendizaje como a partir de los resultados de la evaluación. Los relatos que se registran a continuación explican, por una parte, la función pedagógica y formativa -que tiene para los entrevistadosretroalimentar y orientar al alumnado y, por otra, la importancia que conceden a la flexibilidad que debería tener el profesorado al momento de realizar los ajustes a su planeación curricular, durante todo el proceso formativo. Un profesor señala: “. ..y si les va muy mal nuevamente vuelvo a pasar el contenido, vuelvo a retroalimentar a los niños, e incluso vuelvo a hacer la prueba si es que le va muy mal a todos" (Entrevista 5), y otro docente agrega: "... hay niños que no me comprendieron y yo tengo que volver a retroalimentar de nuevo para poder querer que el niño le quede claro, porque a lo mejor con la actividad que yo tomé no bubo claridad". (Entrevista 9)

La tercera y última demanda expresada por el profesorado que participó en el estudio, en orden a formar al profesorado para evaluar en diversidad de contextos da cuenta de la importancia que asignan a conocer y valorar su saber pedagógico. Al respecto, el profesorado refiere la imperiosa necesidad de diversificar la formación inicial de profesores en evaluación, conociendo el saber pedagógico evaluativo del profeso- 
rado, que ha sido construido en la pluralidad del contexto educativo existente y no sólo aquellos formatos que muestran una evaluación estándar, que no se hace cargo o hace invisible las características particulares de los estudiantes.

A propósito de la realidad que les corresponde vivir en su escuela, el profesorado ejemplifica esta necesidad, a partir de sus propias vivencias. Señalan que en su Escuela no solo deben lidiar con la vulnerabilidad de sus estudiantes, sino que también con el alto número de estudiantes de ascendencia Aymará que concurren a sus clases, grupo ancestral que tiene concepciones del mundo, la naturaleza y la humanidad, muy diferentes a la cultura chilena dominante. Y que, de acuerdo con el relato de los propios profesores han debido incorporar en sus clases. Con pasión y respeto relatan que han aprendido e incorporado en sus relatos de clase algo de la cosmovisión Aymará, que ordena el mundo en tres ámbitos: relaciones sociales, relaciones con divinidades y antepasados y relaciones con la naturaleza. Todo lo cual les ha permitido comprender mucho más la forma de relacionarse y les ha dado la oportunidad de lograr más y mejores aprendizajes.

Señalan como otro rasgo distintivo y positivo de sus espacios laborales, que los estudiantes Aymará tienen habilidades matemáticas superiores a los niños que no tienen esa ascendencia. Esto se explicaría porque están acostumbrados, desde pequeños, a trabajar con sus padres, administrar sus tierras, comercializar los productos que generan en sus huertos y especialmente, a manipular dinero, por lo que la experiencia con las transacciones económicas, desde muy temprana edad, les facilitaría el desarrollo de esas habilidades.

Ahondan en sus respuestas, señalando que las estrategias de evaluación que han implementado atienden esta diversidad, a pesar de no haber sido formados para atender estos contextos escolares:

Entonces claro, un profesor que viene a trabajar en un contexto indígena..., tiene que saber mínimamente lo básico para este tipo del pueblo y los problemas que se va a encontrar. Cómo detecta esto, si él no sabe si el niño es Aymará hablante o no es Aymará hablante, lo único que dice es este niño habla mal, este niño no pronuncia bien, este niño en lenguaje le va pésimo, pero en matemática le va regio. (Entrevista 6) 
No obstante lo anterior y más allá de las evidentes tensiones expresadas en sus relatos, su motivación sustancial es develada con claridad y sin titubeos, al momento de explicar la importancia y la necesidad de brindar una formación educativa capaz de aportar a superar el círculo de vulnerabilidad escolar de sus estudiantes, como se expresa en el siguiente relato:

Todo el rato, nunca dejo de evaluar, porque siempre les estoy diciendo mira, "mírate aquî", "fijate en esto", "cómo está esto con relación a esto", "mira, compáralo con esto que hiciste antes", entonces uno está evaluando todo el tiempo, todo el tiempo, no puedo dejar de hacerlo, la verdad que no puedo dejar de hacerlo si quiero que los niños aprendan. En todo momento uno puede estar evaluando a sus alumnos y generalmente cuando se trata de actividades sencillas una de las actividades que hago como forma de evaluar es evaluarlos oralmente, incluso en ocasiones cuando ellos ni siquiera se dan cuenta. (Entrevista 3)

Bueno, se evalúa prácticamente todos los días, diariamente porque uno va preguntando si aprendieron esto, lo entendieron, lo comprendieron para hacer el cierre porque generalmente a veces uno se olvida, se olvida del cierre, pero yo trato de que no se me olvide, entonces voy preguntando, se entendió el contenido, el concepto, entonces ahí voy evaluando si lo entendieron bien, si no lo entendieron ya vuelvo a repasar antes de la prueba. (Entrevista 2)

\section{Discusión de resultados}

Al examinar los resultados, observamos que los profesores participantes han dado pasos significativos en su hacer profesional, especialmente, al avanzar desde una homogénea formación inicial docente a la construcción, en el aula, de un valioso y poco conocido, y menos valorado, saber pedagógico en evaluación. El profesorado está consciente que este proceso de transición ha implicado la construcción laboriosa de un saber pedagógico evaluativo propio, fundado a partir de una contradicción entre una formación inicial en evaluación homogénea y desarticulada de la realidad de vulnerabilidad que viven cotidiana- 
mente sus estudiantes y su propia práctica evaluativa pedagógica, que ha incorporado con el tiempo las características, habilidades y necesidades particulares de sus estudiantes (Moreno, 2017).

En este importante proceso de transición, lo expresado por el profesorado concuerda con Vallejo y Molina (2014) en dos ámbitos. Primero en la necesidad de contrastar sus propios procesos formativos con su experiencia de aula. Segundo, en la necesidad de vincular su praxis evaluativa con aspectos sustantivos de la vida de sus estudiantes, promoviendo una evaluación más acorde a sus requerimientos y en especial, al contexto escolar de los mismos (Hernández, 2017).

Advertimos que parte de los cambios experimentados por el profesorado, han sido repensar sus prácticas evaluativas, las que en un inicio eran homogéneas y carentes de considerar aprendizajes diferentes al cognitivo, para adaptarlas a las necesidades de sus estudiantes. De tal manera que la investigación realizada, nos permite encontramos con profesores que si bien evalúan a través de procedimientos conocidos por el común de ellos; trabajos prácticos, disertaciones y dramatizaciones, lo hacen porque incentivan la reflexión grupal y personal, con especial énfasis en desarrollar la capacidad de crítica del estudiantado, frente al trabajo personal y el de sus compañeros.

Emerge con fuerza una mayor valoración de aquellas prácticas evaluativas que les permiten, a partir de sus resultados, tomar más y mejores decisiones, y principalmente, implementar procedimientos de evaluación que promueven la opinión crítica del alumnado sobre el desempeño colectivo, sus propios resultados y el de los pares. Es decir, se devela de sus relatos la idea, según la cual, resulta más importante intencionar cómo aplican los instrumentos y qué beneficios reportan a sus estudiantes, que decidir qué instrumento o procedimiento evaluativo usar.

También resulta interesante la valoración que hace el profesorado sobre la importancia del compromiso de los educandos con sus propias tareas de aprendizaje, coincidiendo con Arguello, Filian, Guevara y Cadena (2017), en que las escuelas también necesitan desarrollar en sus estudiantes tanto sus capacidades como sus sentimientos y convicciones. Esta idea se hace evidente, ya que el profesorado considera dentro de sus prácticas evaluativas, promover el trabajo colaborativo 
(Rosseli, 2016), el diálogo, la autoafirmación personal y el desarrollo de habilidades para la vida de sus estudiantes.

Otro de los importantes cambios experimentados por el profesorado ha sido desarrollar sus propias habilidades emocionales, aprendiendo -según sus propias palabras- a ser más humanos y empáticos con sus estudiantes. El saber pedagógico que han construido ha viabilizado la generación de significativas reflexiones respecto a la importancia que poseen los recursos afectivos en la disposición de los estudiantes para aprender y ser evaluados. Tal como lo menciona Ayala y González (2017), los docentes son conscientes del beneficio de favorecer el aprendizaje y mejorar los resultados de la evaluación, incorporando la expresión constante de palabras y gestos afectuosos, como elementos mediadores y generadores de diálogo con los estudiantes y entre los estudiantes.

Vinculado a la incorporación de los recursos afectivos en sus prácticas evaluativas, la constante construcción del saber pedagógico les ha permitido distinguir la relevancia de realizar la acción docente desde las altas expectativas (Moneo y Huertas, 2017), que se evidencian -según sus relatos- en explicitar verbal y gestualmente al alumnado que todos pueden, sin distinción, aprender y conseguir las metas que se planteen.

Respecto de los requerimientos a la formación inicial docente, tenemos la convicción de que sus procesos formativos no han sido del todo pertinentes ni satisfactorios. Si bien critican la formación recibida en la educación superior, argumentando que desconoce los diferentes contextos escolares y no logra sintonizar con los desafíos reales que posee el profesorado en servicio, no logran avanzar hacia una autocrítica de su responsabilidad profesional en el proceso, ni menos cuestionar su manejo del contenido disciplinar y de la didáctica asociada (Ibáñez, 2014).

Desde su saber pedagógico, el profesorado hace diversas sugerencias a la formación inicial de profesores, cuyas ideas relevantes se centran en tres ámbitos: i) formar al profesorado para evaluar constantemente, ii) formar al profesorado para retroalimentar después de evaluar y iii) formar al profesorado para evaluar en diversidad de contextos. Fruto de su experiencia en evaluación, describen el proceso evaluativo 
como una constante y variada recogida de información, aunque al ser consultados por las calificaciones y su importancia, hace que el profesorado tensione su discurso y transite entre un rol tradicional y un rol de mediador y facilitador de diálogos con y entre los alumnos, como efectivamente debería ser (Rittaco y Amores, 2016).

\section{Conclusiones}

El profesorado que se desempeña en escuelas vulnerables que participó en el estudio, ha dado pasos significativos en su hacer profesional, avanzando desde una homogénea formación inicial docente a la construcción, en el aula, de un valioso saber pedagógico en evaluación. Queda en evidencia que este proceso de transición ha implicado que el saber pedagógico se ha ido modificando en consideración de las características de los estudiantes y del contexto escolar vulnerable donde se desempeñan.

De allí que los resultados muestren los cambios que han experimentado en sus prácticas evaluativas, reconozcan los aportes de la evaluación al aprendizaje de los estudiantes y elaboren recomendaciones a la academia, desde su saber pedagógico, respecto de la formación inicial de profesores en el ámbito de la evaluación.

Los resultados de este estudio evidencian cómo la construcción del saber pedagógico evaluativo se ha ido modificando en función de la experiencia del profesorado en esta materia. De tal manera que no cabe dudas de la plasticidad del profesorado para adecuarse y atender a las características y necesidades de sus estudiantes, incorporando en sus prácticas una evaluación más holística, con un sentido integrador, pertinente y centrada en el alumnado.

Resulta muy interesante advertir la importancia que atribuye el profesorado a los recursos afectivos como elemento mediador y facilitador de aprendizaje. Relevan el buen trato, la empatía y el diálogo atento y fraterno, como parte de las acciones que emprenden intencionadamente en este ámbito. Fruto de este cambio, han generado un quehacer diario más amable, que trasciende a un espacio de aula más grato, acogiendo a los alumnos y validando sus emociones en función 
del aprendizaje con sentido. Para avanzar en este sentido, el profesorado debió cambiar su manera tradicional de enfrentar las relaciones interpersonales y repensar los modos -distantes y despersonalizadosen que se vinculaban con el alumnado, estilos que, por supuesto, nunca fueron enseñados en la formación inicial.

Tan interesante como el punto anterior son las demandas y recomendaciones que realizan desde su saber pedagógico en evaluación a las instituciones de educación superior que forman, descontextualizadamente, a los futuros profesores. Los desafían a conocer, analizar y visibilizar las prácticas evaluativas del profesorado y principalmente, acompañar a los profesores en formación en el ajuste entre el saber pedagógico en evaluación y lo que realmente realizan en el aula.

Este estudio corrobora lo señalado por la OCDE (2011), respecto de que los profesores en formación tienen una enseñanza homogénea y desvinculada de los diversos contextos escolares. Será un reto para la formación inicial docente entonces, atender las demandas del profesorado en ejercicio, valorando su saber pedagógico y retroalimentando su itinerario formativo desde esa experiencia.

\section{Referencias}

Abraham, M. (2017). Pedagogía, saber pedagógico y prácticas educativas: reflexiones sobre una experiencia. Revista de pedagogía crítica Paulo Freire, 7, 119-131

Arguello, L., Filian, F., Guevara, J. C. y Cadena, L. (2017). La importancia de la determinación de indicadores de desarrollo. La unidad de lo afectivo y lo cognitivo. Revista Didasc@lia: Didáctica y Educación, 8, 193-206.

Ayala, M., y González, J. A. (2017). Las competencias socio-afectivas docentes y la formación para la práctica educativa del desarrollo personal y para la convivencia, en el marco de la educación inclusiva. Revista de Educación Inclusiva, 8, 271-284.

Bonilla, M. Á., y López, A. D. (2016). Ejemplificación del proceso metodológico de la teoría fundamentada. Cinta de Moebio: Revista de Epistemología de Ciencias Sociales, 57, 305-315.

Casassus, J. (2017). Una introducción a la Educación Emocional. Revista Latinoamericana de Politicas y Administración de la Educación, 7, 121-130. 
Cubillos, J. (2018). El significado de las competencias emocionales para Educadoras de Párvulos. Paideia, Revista de Educación, 62, 81-106.

Ministerio de Educación (2018). Docente Más / Sistema de Evaluación Docente de Chile. Recuperado de: https:/www.docentemas.cl/pages/ conozca-la-evaluacion/marco-legal.php

Dorio, I.; Sabariego, M. y Massot, I. (2016). Métodos de Investigación Cualitativa. En Bisquerra, R. (coord.) Metodología de la investigación educativa (pp. 285-320). Madrid: Editorial: La Muralla S.A.

Espínola, V., Treviño, J. E., Guerrero, M., y Martínez, J. (2017). Liderazgo para la mejora en escuelas vulnerables: prácticas asociadas al cumplimiento de metas de aprendizaje. Estudios Pedagógicos, 43, 87-106.

Flick, U. (2018). An introduction to qualitative research. Los Angeles: Sage Publications Limited.

Gairín, J., y Sanmartí, N. (2010). Evaluación del proceso formativo y del aprendizaje. Madrid: Universidad de Alcalá. Instituto Sindical de Cooperación al Desarrollo. Master en Formación de Formadores Sociolaborales.

Glaser, B. G., \& Strauss, A. L. (2009). The Discovery of Grounded Theory: Strategies for Qualitative Research. New Jersey: Transaction Publishers.

Gómez, G., \& Rivas, M. (2017). Resiliencia académica, nuevas perspectivas de interpretación del aprendizaje en contextos de vulnerabilidad social. Calidad en la educación, 47, 215-233. Recuperado de: https://scielo.conicyt.cl/scielo.php?pid=S0718-45652017000200215\&script=sci_arttext

Hernández, M. (2017). ¿Por qué ha costado tanto transformar las prácticas evaluativas del aprendizaje en el contexto educativo? Ensayo crítico sobre una patología pedagógica pendiente de tratamiento. Revista Electrónica Educare, 21(1), 12. Recuperado de: https://dialnet.unirioja.es/servlet/ articulo?codigo $=5763957$

Hernández, R., Fernández, C., \& Baptista, P. (2010). Metodología de la investigación. México, DF.

Ibáñez, N. (2014). Saberes profesionales para la transformación del modelo educativo en Chile. Revista Estudios Pedagógicos, 40 (Especial), 145160. Recuperado de https://scielo.conicyt.cl/scielo.php?pid=S071807052014000200009\&script=sci_arttext

Infante, M., Matus, C., Paulsen, A., Salazar, A., \& Vizcarra, R. (2013). Narrando la vulnerabilidad escolar: Perfomatividad, espacio y territorio. $\mathrm{Li}$ teratura y Lingüistica, 27, 281-308. Recuperado de: https://scielo.conicyt. c1/scielo.php?pid=S0716-58112013000100014\&script=sci_arttext

Jiménez-Fontana, R., García-González, E., Azcárate, P., Navarrete, A., \& Cardeñoso, J. M. (2016). La Teoría Fundamentada como estrategia de 
análisis de los datos: caracterización del proceso. In Atas del $5^{\circ}$ Congreso Iberoamericano em Investiaçao Qualitativa, Vol. 1 (pp. 356-365). Portugal: CIAIQ. Recuperado de: https://proceedings.ciaiq.org/index.php/ ciaiq2016/article/view/619

JUNAEB (2005). SINAE, Sistema Nacional de Asignación con Equidad para Becas JUNAEB: Una nueva visión en la construcción de igualdad de oportunidades en la infancia. Recuperado de: https://www.junaeb.cl/wpcontent/uploads/2013/02/libro_junaeb.pdf

Longás, J., Cussó, I., De Querol, R., \& Riera, J. (2016). Análisis de factores de apoyo a trayectorias de éxito escolar en la enseñanza secundaria en contextos de pobreza y vulnerabilidad social en España. Un estudio de casos múltiples. Rexe. Revista de Estudios y Experiencias en Educación, 15(28), 107-127). Recuperado de: http://www.rexe.cl/ojournal/index. $\mathrm{php} / \mathrm{rexe} /$ article/view/21

López, N., \& Sandoval, I. (2016). Métodos y técnicas de investigación cuantitativa y cualitativa. Recuperado de: http://biblioteca.udgvirtual. udg.mx/jspui/bitstream/123456789/176/3/M\%C3\%A9todos\%20y\%20 t\%C3\%A 9cnicas\%20de\%20investigaci\%C3\%B3n\%20cuantitativa\%20 y\%20cualitativa.pdf

López-Lozano, L., Solís, E., \& Fernández,J. (2017). Los cambios en las concepciones sobre evaluación en ciencias de futuros maestros de Primaria. Revista Complutense de Educación, 29(3), 847-864. Recuperado de https:// idus.us.es/xmlui/handle/11441/77550

Martínez, B. H. (2015). Causas del bajo rendimiento escolar origina un alto nivel de deserción escolar y habilidades para estudiar ayudan a mejorar el rendimiento escolar. Revista Iberoamericana para la Investigación y el Desarrollo Educativo, 11, 11-17.

Moneo, M., \& Huertas, J. (2017). Motivación y cambio conceptual. Tarbiya, Revista de Investigación e Innovación Educativa, 26, 51-71. Recuperado de https://revistas.uam.es/tarbiya/article/view/7115

Monereo, C. y Barberá, E. (2000). Diseño instruccional de las estrategias de aprendizaje en entornos educativos no-formales. En Monereo et al. Estrategias de aprendizaje. Madrid: Visor/Ediciones de la Universitat Oberta de Catalunya. Recuperado de: https://dialnet.unirioja.es/servlet/ articulo? codigo $=988809$

Monge, V. (2015). La codificación en el método de investigación de la Grounded Theory o Teoría Fundamentada. Revista Innovaciones Educativas, 17(22), 77-84. Recuperado de https://dialnet.unirioja.es/servlet/ articulo?codigo $=5248462$ 
Moreno, T. (2017). La evaluación ¿̇nos conducirá a la tierra prometida? Perspectiva Educacional, 56(1), 147-163. Recuperado de http://www.perspectivaeducacional.cl/index.php/peducacional/article/view/453

Murillo, F. J., \& Martínez-Garrido, C. (2018). Factores de aula asociados al desarrollo integral de los estudiantes: Un estudio observacional. Estudios pedagógicos, 44(1), 181-205. Recuperado de: https://scielo.conicyt.cl/scielo.php?pid=S0718-07052018000100181\&script=sci_arttext

Nanculeo, M. A., \& Merino, J. M. (2016). Una aproximación a la vulnerabilidad en el sistema de educación parvularia en Chile. Nóesis. Revista de Ciencias Sociales y Humanidades, 25(50), 51-88. Recuperado de: https:// www.redalyc.org/pdf/859/85944887003.pdf

Nanculeo, M. A. (2014). Construcción de un índice de vulnerabilidad social para estudiantes preescolares de establecimientos subvencionados de Chile (Disertación doctoral, Universidad de Concepción, Facultad de Ciencias Sociales, Departamento de Sociología). Recuperado de: http:// repositorio.udec.c1/handle/11594/1572

OECD (2011). Panorama de la educación 2010. Indicadores de la OCDE. Ministerio de Educación.

Rittaco, M., \&Amores, F.J. (2016). Percepciones del profesorado y alumnado del programa de cualificación profesional inicial (PCPI). Desarrollo del proceso de enseñanza-aprendizaje en programas de prevención del fracaso escolar en secundaria. Espiral. Cuadernos del profesorado, 9(18), 3-15. Recuperado de https://dialnet.unirioja.es/servlet/articulo?codigo $=5428047$

Rof, J. (1975). Fronteras vivas del psicoanálisis: conferencias organizadas por el Instituto de Ciencias del Hombre. Madrid, España: Karpos.

Roselli, N. D. (2016). El aprendizaje colaborativo: Bases teóricas y estrategias aplicables en la enseñanza universitaria. Propósitos y Representaciones, 4(1), 219-280. Recuperado de: http://revistas.usil.edu.pe/index.php/pyr/ article/view/90

Sabino, C. (2014). El proceso de investigación. Editorial Episteme.

San Martín, D. (2014). Teoría fundamentada y Atlasti: recursos metodológicos para la investigación educativa. Revista Electrónica de Investigación Educativa, 16(1), 103-122. Recuperado de: http://www.scielo.org.mx/ scielo.php?script=sci_arttext\&pid=S1607-40412014000100008

Sánchez, G., Díaz, S., Fuentes, A., \& Osorio, J. (2017). Representaciones sobre el ejercicio de la docencia en contextos de vulnerabilidad de profesores de ciencias sociales en práctica. Recuperado de: http://rephip.unr. edu.ar/handle/2133/12915

Solar, M. I., \& Díaz, C. (2018). El profesor universitario: construcción de 
su saber pedagógico e identidad profesional a partir de sus cogniciones y creencias. Calidad en la Educación, 30, 208-232. Recuperado de https:// calidadenlaeducacion.cl/index.php/rce/article/view/178

Sotos, M., \& López, M. C. (2015). El proceso de construcción del saber pedagógico en Educación Matemática: el caso de María Antònia Canals. Epsilon: Revista de la Sociedad Andaluza de Educación Matemática Thales, 90, 59-69. Recuperado dehttp://thales.cica.es/epsilon/sites/thales.cica. es.epsilon/files/\%5Bfield_volumen-formatted\%5D/epsilon90_6.pdf

Strauss, A. L., Corbin, J., \& Zimmerman, E. (2002). Bases de la investigación cualitativa: técnicas y procedimientos para desarrollar la teoría fundamentada. Medellín: Universidad de Antioquia.

Suárez, D. (2017). Docentes, relatos de experiencia y saberes pedagógicos. La documentación narrativa de experiencias en la escuela. Investigación Cualitativa, 2(1), 42-54. Recuperado de: https://ojs.revistainvestigacioncualitativa.com/index.php/ric/article/view/58

Traverso, C. V. (2016). Hacia la innovación en la formación inicial docente para un desempeño exitoso en contextos alta vulnerabilidad social y educativa. Rexe. Revista de Estudios y Experiencias en Educación, 12(23), 4759. Recuperado de: http://www.rexe.cl/ojournal/index.php/rexe/article/ view/78

Tucker, S., Trotman, D., \& Martyn, M. (2015). Vulnerability: The role of schools in supporting young people exposed to challenging environments and situations. International Journal of Educational Development, 41, 301306. Recuperado de: https://www.sciencedirect.com/science/article/pii/ S0738059314000972

Vallejo, M., \& Molina, J. (2014). La evaluación auténtica de los procesos educativos. Revista Iberoamericana de educación Educativa, 64, 11-25. Recuperado de http://www.academia.edu/download/36950574/evaluacion_2.pdf 


\section{Appendix 1: Instrument after alternative assessment procedure}

\section{Efectos de distintos tipos de evaluación}

La siguiente encuesta tiene como propósito contrastar los efectos que producen dos tipos de evaluaciones en los estudiantes. La información entregada tiene fines estrictamente académicos; por lo tanto, se asegura confidencialidad.

No hay respuestas correctas, ni incorrectas. La encuesta consta de dos secciones: la primera, de carácter biográfico y la segunda relacionada con los efectos de la evaluación. Se valora su mayor honestidad al momento de responder las preguntas. La encuesta es de carácter voluntario, por lo que puede desistir de hacerla en cualquier momento.

I. Encierre la respuesta que más se acerca a su opinión. Encierre sólo una respuesta.

1. Considero que mi nivel de inglés es:
a) Muy bueno
b) Bueno
c) Regular
d) Malo

2. Mi interés por aprender inglés es:
a) Alto
b) Regular
c) Bajo
d) No tengo interés

3. Creo que aprender inglés es:
a) Muy importante
b) Más o menos importante
c) No tan importante
d) No tiene importancia

4. Creo que aprendo mejor a través de:

a) Evaluaciones prácticas (por ejemplo, presentaciones orales, creación de álbumes, etc.)

b) Pruebas escritas

Su nombre es confidencial y será utilizado solo para comparar los resultados de las diferentes encuestas.

Nombre:

¡Muchas gracias! 


\section{Marque con una "X" su respuesta.}

MA: Muy de acuerdo

A: De acuerdo

D: En desacuerdo

MD: Muy en desacuerdo

\begin{tabular}{|c|c|c|c|c|c|}
\hline & & $\begin{array}{c}\text { MA } \\
1\end{array}$ & $\begin{array}{l}\text { A } \\
2\end{array}$ & $\begin{array}{l}\mathrm{D} \\
3\end{array}$ & $\begin{array}{c}\mathrm{MD} \\
4\end{array}$ \\
\hline 1 & $\begin{array}{l}\text { Me sentí motivada/o a trabajar en el tema/contenido de esta } \\
\text { evaluación. }\end{array}$ & & & & \\
\hline 2 & Disfruté estudiando/trabajando para esta evaluación. & & & & \\
\hline 3 & $\begin{array}{l}\text { Dediqué más tiempo para preparar esta evaluación porque lo } \\
\text { disfruté. }\end{array}$ & & & & \\
\hline 4 & Esta evaluación fue relevante para mejorar mi uso del inglés. & & & & \\
\hline 5 & El tema/contenido me motivó a averiguar al respecto. & & & & \\
\hline 6 & $\begin{array}{l}\text { Mi interés sobre el tema abordado en la evaluación aumentó al } \\
\text { realizar esta medición. }\end{array}$ & & & & \\
\hline 7 & Soy capaz de motivarme para mejorar. & & & & \\
\hline 8 & Me sentía nerviosa/o antes de la evaluación. & & & & \\
\hline 9 & $\begin{array}{l}\text { La importancia que tiene esta evaluación en mis notas, influyó } \\
\text { en mi desempeño. }\end{array}$ & & & & \\
\hline 10 & $\begin{array}{l}\text { Creo que con este tipo de evaluación puedo demostrar mis } \\
\text { conocimientos del idioma inglés sin estresarme mucho }\end{array}$ & & & & \\
\hline 11 & Me siento cómoda/o realizando este tipo de evaluación. & & & & \\
\hline 12 & $\begin{array}{l}\text { Me siento segura/o de mis conocimientos en este tipo de } \\
\text { evaluación. }\end{array}$ & & & & \\
\hline 13 & $\begin{array}{l}\text { Estaba nerviosa/o por el tiempo que tuve para realizar la } \\
\text { evaluación. }\end{array}$ & & & & \\
\hline 14 & Me sentía preparada/o al momento de hacer la evaluación. & & & & \\
\hline 15 & $\begin{array}{l}\text { Recibí retroalimentación constante durante esta evaluación lo } \\
\text { que disminuyó mi estrés por la nota. }\end{array}$ & & & & \\
\hline 16 & $\begin{array}{l}\text { Utilicé diferentes estrategias para resolver problemas de último } \\
\text { minuto. }\end{array}$ & & & & \\
\hline 17 & Organicé bien mi tiempo para poder terminar en forma exitosa. & & & & \\
\hline 18 & $\begin{array}{l}\text { Usé diferentes habilidades en esta evaluación para aprender } \\
\text { mejor. }\end{array}$ & & & & \\
\hline 19 & $\begin{array}{l}\text { Leí atentamente las instrucciones antes de comenzar esta } \\
\text { evaluación. }\end{array}$ & & & & \\
\hline 20 & $\begin{array}{l}\text { Las estrategias de aprendizaje que poseo fueron útiles para } \\
\text { realizar esta evaluación. }\end{array}$ & & & & \\
\hline
\end{tabular}




\section{Appendix 1: Instrument after the traditional test}

\section{Efectos de distintos tipos de evaluación}

La siguiente encuesta tiene como propósito contrastar los efectos que producen dos tipos de evaluaciones en los estudiantes. La información entregada tiene fines estrictamente académicos; por lo tanto, se asegura confidencialidad.

No hay respuestas correctas, ni incorrectas. La encuesta consta de dos secciones: la primera, de carácter biográfico y la segunda relacionada con los efectos de la evaluación. Se valora su mayor honestidad al momento de responder las preguntas. La encuesta es de carácter voluntario, por lo que puede desistir de hacerla en cualquier momento.

I. Encierre la respuesta que más se acerca a su opinión. Encierre sólo una respuesta.

1. Considero que mi nivel de inglés es:
b) Muy bueno
b) Bueno
c) Regular
d) Malo

2. Mi interés por aprender inglés es:
a) Alto
b) Regular
c) Bajo
d) No tengo interés

3. Creo que aprender inglés es:
a) Muy importante
b) Más o menos importante
c) No tan importante
d) No tiene importancia

4. Creo que aprendo mejor a través de:

a) Evaluaciones prácticas (por ejemplo, presentaciones orales, creación de álbumes, etc.)

b) Pruebas escritas

Su nombre es confidencial y será utilizado solo para comparar los resultados de las diferentes encuestas.

Nombre:

¡Muchas gracias! 


\section{Marque con una "X" su respuesta.}

MA: Muy de acuerdo

A: De acuerdo

D: En desacuerdo

MD: Muy en desacuerdo

\begin{tabular}{|c|c|c|c|c|c|}
\hline & & \multirow[b]{2}{*}{$\begin{array}{c}\text { MA } \\
1\end{array}$} & \multirow[b]{2}{*}{$\begin{array}{l}A \\
2\end{array}$} & \multirow{3}{*}{$\begin{array}{l}\mathrm{D} \\
3\end{array}$} & \multirow{3}{*}{$\begin{array}{c}\mathrm{MD} \\
4\end{array}$} \\
\hline & & & & & \\
\hline 1 & $\begin{array}{l}\text { Las estrategias de aprendizaje que poseo fueron útiles para realizar } \\
\text { esta evaluación. }\end{array}$ & & & & \\
\hline 2 & $\begin{array}{l}\text { Me sentí motivada/o a trabajar en el tema/contenido de esta } \\
\text { evaluación. }\end{array}$ & & & & \\
\hline 3 & Esta evaluación fue relevante para mejorar mi uso del inglés. & & & & \\
\hline 4 & $\begin{array}{l}\text { Me siento segura/o de mis conocimientos en este tipo de } \\
\text { evaluación. }\end{array}$ & & & & \\
\hline 5 & $\begin{array}{l}\text { Utilicé diferentes estrategias para resolver problemas de último } \\
\text { minuto. }\end{array}$ & & & & \\
\hline 6 & Usé diferentes habilidades en esta evaluación para aprender mejor. & & & & \\
\hline 7 & $\begin{array}{l}\text { La importancia que tiene esta evaluación en mis notas, influyó en } \\
\text { mi desempeño. }\end{array}$ & & & & \\
\hline 8 & $\begin{array}{l}\text { Creo que con este tipo de evaluación puedo demostrar mis } \\
\text { conocimientos del idioma inglés sin estresarme mucho. }\end{array}$ & & & & \\
\hline 9 & Me sentía preparada/o al momento de hacer la evaluación. & & & & \\
\hline 10 & Disfruté estudiando/trabajando para esta evaluación. & & & & \\
\hline 11 & $\begin{array}{l}\text { Leí atentamente las instrucciones antes de comenzar esta } \\
\text { evaluación. }\end{array}$ & & & & \\
\hline 12 & Me siento cómoda/o realizando este tipo de evaluación. & & & & \\
\hline 13 & $\begin{array}{l}\text { Recibí retroalimentación constante durante esta evaluación lo que } \\
\text { disminuyó mi estrés por la nota. }\end{array}$ & & & & \\
\hline 14 & Soy capaz de motivarme para mejorar. & & & & \\
\hline 15 & $\begin{array}{l}\text { Estaba nerviosa/o por el tiempo que tuve para realizar la } \\
\text { evaluación. }\end{array}$ & & & & \\
\hline 16 & El tema/contenido me motivó a averiguar al respecto. & & & & \\
\hline 17 & Organicé bien mi tiempo para poder terminar en forma exitosa & & & & \\
\hline 18 & $\begin{array}{l}\text { Dediqué más tiempo para preparar esta evaluación porque lo } \\
\text { disfruté. }\end{array}$ & & & & \\
\hline 19 & Me sentía nerviosa/o antes de la evaluación. & & & & \\
\hline 20 & $\begin{array}{l}\text { Mi interés sobre el tema abordado en la evaluación aumentó al } \\
\text { realizar esta medición. }\end{array}$ & & & & \\
\hline
\end{tabular}

